\title{
Efficacy of Mucosal Cutting Biopsy for the Histopathological Diagnosis of Gastric Submucosal Tumors
}

\author{
Akihisa Adachi $^{\mathrm{a}}$ Yoshikazu Hirata ${ }^{\mathrm{a}}$ Hayato Kawamura ${ }^{\mathrm{a}}$ \\ Takahito Harada $^{a}$ Reika Hattori ${ }^{a}$ Daisuke Kumai ${ }^{a}$ Yuki Yamamoto ${ }^{a}$ \\ Yuki Kojima ${ }^{a}$ Hirokazu Ikeuchi ${ }^{a}$ Noriyuki Hayashia Hisato Mochizuki ${ }^{a}$ \\ Hiroki Takada ${ }^{a}$ Ryuzo Yamaguchib Satoshi Sobue ${ }^{a}$ \\ aDepartment of Gastroenterology, Kasugai Municipal Hospital, Kasugai, Japan; \\ bDepartment of Surgery, Kasugai Municipal Hospital, Kasugai, Japan
}

\section{Keywords}

Submucosal tumor - Endoscopic ultrasound-guided fine needle aspiration - Mucosal cutting biopsy

\begin{abstract}
Background: Gastrointestinal stromal tumors occur frequently. Endoscopic ultrasound-guided fine needle aspiration (EUS-FNA) is performed commonly for diagnosis. However, the success rate of histological diagnosis is insufficient when the submucosal tumor (SMT) is small. Recently, another technique, mucosal cutting biopsy (MCB) has been reported. The aim of this study is to evaluate the efficacy and safety of MCB. Method: Between January 2012 and August 2018, MCB and EUS-FNA were performed 16 and 31 times for diagnosing gastric SMT. The diagnostic rate, the rate of successful immunohistochemistry, and the safety were reviewed. Difficult locations for EUS-FNA were also evaluated. Results: The mean SMT sizes measured on MCB and EUS-FNA were 21.2 and $36.2 \mathrm{~mm}$. The diagnostic rates of MCB and EUS-FNA were almost the same (88 vs. $81 \%$ ), but successful immunohistochemistry was significantly higher in
\end{abstract}


the MCB group (93 vs. 59\%, $p=0.03$ ). In the subgroup of SMTs $<20 \mathrm{~mm}$, the successful histological diagnosis rate from EUS-FNA was relatively low. There were no complications. Failures of EUS-FNA were more frequent in the middle third of the stomach. Conclusions: MCB was an effective procedure for diagnosing gastric SMT, especially in the case of small SMTs located at the middle third of the stomach.

\section{Introduction}

Gastrointestinal stromal tumors (GISTs) are the most frequent submucosal tumors (SMTs) of the stomach. The Japanese GIST Therapeutic Guideline [1] states that surgical resection is indicated if a histopathological diagnosis of GIST is made, because GIST has malignant potential even if it is not very large. The diagnosis of SMT including GIST is made by immunostaining with c-kit, CD34, SMA, S100, and so on. Generally, in cases where positive staining for c-kit or CD34 and negative staining for SMA and S-100 is confirmed, the tumor is diagnosed as GIST. So, immunostaining is very important for the diagnosis of SMT. But immunostaining is often difficult when the amount of obtained specimen is small.

Endoscopic ultrasound-guided fine needle aspiration (EUS-FNA) is reported to be the most useful method to obtain specimens from SMTs [1-3]. The rate of successful diagnosis of SMTs by EUS-FNA combined with cytology has been reported to be relatively high (83\%), but the rate of successful diagnosis by histology is not satisfactory (50\%) [4-7]. With respect to tumor size, many reports showed that the diagnostic rate was insufficient in SMTs less than 2 $\mathrm{cm}$ in diameter $[8,9]$. However, several papers reported that small SMTs (diameter $<2 \mathrm{~cm}$ ) had a high-grade malignant potential, and these tumors progressed rapidly $[10,11]$.

Recently, another diagnostic method for small gastric SMT has been reported, the socalled mucosal cutting biopsy (MCB) technique $[12,13]$. To apply this technique, a relatively large specimen could be obtained under direct view in a short time, and the diagnostic rate including immunostaining was reported to be satisfactorily high, even for small SMTs. Thus, this study was designed to compare the histopathological diagnostic ability including immunohistochemistry of EUS-FNA and MCB for gastric SMTs.

The purpose of this study was to evaluate the efficacy of MCB for the histopathological diagnosis of gastric SMTs and establish a strategy for the diagnosis of gastric SMTs.

\section{Materials and Methods}

\section{Patient Population}

Between January 2012 and August 2018 in the Department of Gastroenterology, Kasugai Municipal Hospital, 40 patients were referred for diagnostic examination of gastric SMT, and all of these patients were included in this study. Based on the Japanese GIST Therapeutic Guidelines, EUS-FNA was chosen in principle as the diagnostic procedure. If insufficient material for histopathological examination was obtained by EUS-FNA or it was judged that EUSFNA was not suitable because of tumor size or location, the MCB procedure was selected to 
obtain the necessary materials for a precise histopathological diagnosis. As a result, MCB and/or EUS-FNA was performed in 40 gastric SMT cases.

MCB alone was performed in 9 patients, and EUS-FNA alone was performed in 24 patients. Both MCB and EUS-FNA were performed in 7 patients. Thus, 16 patients underwent MCB, and 31 patients underwent EUS-FNA (Fig. 1). In this study, the histopathological diagnostic yield, efficacy, and safety of MCB and of EUS-FNA were compared.

\section{Mucosal Cutting Biopsy Procedures}

MCB was performed as previously reported by Kataoka et al. [12]. Briefly, after saline injection into the submucosal layer, mucosal cutting was performed using a needle knife. Under direct vision of the SMT, several biopsy specimens were obtained using biopsy forceps. Then, mucosal incision was closed with clips.

\section{EUS-FNA Procedures}

The EUS-FNA procedure was performed using standard technique. After targeting the mass using a convex linear echoendoscope (GF-UCT 260; Olympus, Tokyo, Japan), fine needle aspiration was performed using a 22-gauge needle (Expect Endoscopic Ultrasound Aspiration Needle; Boston Scientific, Marlborough, MA, USA). The needle size was changed according to the tumor size, rigidity, and location, as appropriate.

The obtained specimen was submitted to rapid on-site evaluation (ROSE) and FNA procedure was completed after the positive report of ROSE.

\section{Analysis Parameters}

The histopathological diagnostic yield and the rate of successfully obtaining a biopsy specimen for histopathological examination including immunohistochemistry were compared between MCB and EUS-FNA. The relationship between SMT size and the diagnostic rate was evaluated in each group. The location of each SMT relevant to diagnostic yield was also evaluated in the EUS-FNA group.

\section{Statistical Analysis}

Continuous variables are expressed as mean values \pm SD (standard deviation) and compared between two groups using Welch's $t$ test. Categorical variables were compared using the chi-squared test. Fisher's exact test was applied as appropriate. All statistics were calculated using R software, version 3.4.3 (R Development Core Team, 2017). Probabilities of less than 0.05 were considered significant.

\section{Results}

\section{Patients' Characteristics}

The characteristics of the 40 cases are summarized in Table 1 . There were no significant differences in mean age and sex ratio between the two groups. There were clearly more SMTs less than $2 \mathrm{~cm}$ in the MCB group ( $p=0.02$ ). Extraluminal SMTs tended to be more common in the EUS-FNA group $(p=0.09)$. Most cases of SMT originated from the muscularis propria in both groups. 


\section{Case Reports in Gastroenterology}

Case Rep Gastroenterol 2019;13:185-194

DOI: $10.1159 / 000499442$

(c) 2019 The Author(s). Published by S. Karger AG, Basel www.karger.com/crg

Adachi et al.: Efficacy of Mucosal Cutting Biopsy for the Histopathological Diagnosis of Gastric Submucosal Tumors

\section{Comparison between MCB and EUS-FNA}

Table 2 shows the results of MCB and EUS-FNA. There was no significant difference in procedure time between MCB and EUS-FNA. The rate of successful diagnosis was the same in both groups, but the rate of successful immunohistochemistry was significantly higher in the MCB group. Regarding immunohistochemical staining in the MCB group, in most cases in which adequate specimens were obtained, they could be evaluated by immunostaining, while in the EUS-FNA group, the rate of successful immunostaining was only $59 \%(p=0.03)$.

A typical case where both EUS-FNA and MCB were performed is presented in Figure 2. Histopathological examination of EUS-FNA, MCB, and surgical specimen was shown, respectively. The amount of obtained specimen by EUS-FNA was small and as a result, definitive diagnosis was not made because histopathological examination including immunostaining was impossible (Fig. 2a). On the other hand, adequate specimen could be obtained by MCB in the same case. HE staining revealed a lot of spindle cells in muscle layer, and immunostaining was positive for c-kit and CD34 and negative for SMA and S-100 (Fig. 2b). According to these findings, the SMT was diagnosed as GIST. This tumor was resected, and immunostaining of the surgical specimen was completely the same as the MCB specimen (Fig. 2c).

Immunostaining data were evaluated excluding aberrant pancreas, because immunostaining is not needed for diagnosis. In the subgroup of SMTs less than $2 \mathrm{~cm}$, EUS-FNA had low rates of obtaining adequate materials for diagnosis and of successful immunohistochemistry, but because of the small sample size, there were no significant differences. There were no complications in either group (Table 2).

Seven cases in the MCB group were treated surgically, and 24 in the EUS-FNA group were operated, and the surgical specimens were evaluated histopathologically in comparison with the preoperative specimens. Both groups had similar histopathological concordance rates between before and after surgery. All cases of GIST were resected surgically. In the MCB group, one schwannoma was operated because of a large ulcer, and one SMT diagnosed as leiomyoma was operated because another GIST existed in the same patient, but after operation, the leiomyoma was also diagnosed as GIST. In the EUS-FNA group, there were 4 cases that required operation in the "not diagnosed" group. Two cases of GIST and one gastric cancer diagnosed by MCB after unsuccessful EUS-FNA procedures were operated. One schwannoma was also operated because of its large size and lymph node swelling around the stomach (Table 2).

\section{Analysis of EUS-FNA Procedures}

The technical success rate of EUS-FNA was evaluated for each location of gastric SMT (Table 3). In the intraluminal group, unsuccessful procedures were more common in the middle gastric body, but there was no significant difference. On the other hand, EUS-FNA was successful in most extraluminal SMTs.

\section{Discussion}

This was a retrospective study comparing the efficacy of MCB and EUS-FNA. Generally, MCB is performed with a needle knife and biopsy forceps. The technique of MCB is standard, and it is not necessary to prepare special devices. The cost may be less with MCB [14]. This study showed that MCB was not inferior in procedure time, the rate of successful diagnosis, 
and the concordance rate of pathologic findings after operation comparing with EUS-FNA. Moreover, the rate of successful immunostaining was significantly higher with MCB than with EUS-FNA. Immunohistochemistry is an essential examination to distinguish GISTs from leiomyomas, schwannomas, and so on. In addition, in the cases of SMTs less than $2 \mathrm{~cm}$, the diagnostic yield was significantly better in the MCB group. There are some reports that several small GISTs also have malignant potential, and small SMTs may grow faster than large ones $[15,16]$. Considering these facts, it is better to obtain a sufficient SMT specimen, even if the tumor is small. In that case, MCB is an important technique.

EUS-FNA is a very important procedure for the diagnosis of SMT. However, the rate of successful EUS-FNA varies in each hospital because of the need for technical experience. The rate of successful histological diagnosis by EUS-FNA for gastric SMTs is reported to be about $60-80 \%[4,5]$. With respect to small GISTs, the rate of successfully obtaining cytological and histological specimens was reported to be $81.3 \%$ by combined use of ROSE [16]. In the present study, the diagnostic rate by EUS-FNA was in line with other reports, but it was unfavorable in cases less than $2 \mathrm{~cm}$, even though the cases were not so many. Even when specimens could be obtained by EUS-FNA, about half of the cases could not be assessed immunohistologically.

The rate of successful histological diagnosis was evaluated for each area of the stomach in the EUS-FNA group. The rate of unsuccessful EUS-FNA was slightly higher in the middle third of the stomach than other areas, but there was no significant difference. Niimi et al. [17] reported that SMT located in the middle third of the stomach is a predictive factor for nondiagnosis when beginners try to perform EUS-FNA. The present result was in line with Niimi et al. [17]. Thus, MCB may be the first choice when an SMT is less than $2 \mathrm{~cm}$ or it is located in the middle third of the stomach. In fact, in the present study, there were 3 cases in which EUSFNA failed but sufficient materials were obtained by MCB.

EUS-FNA is a relatively safe method. It is reported that adverse events that might be caused by EUS-FNA are bleeding and infection, but they are relatively rare [18]. Regarding the adverse events of the MCB procedure, Kataoka et al. [12] also reported the safety of this technique. In the present study, both groups had no complications. Thus, MCB is considered as safe as EUS-FNA.

There are several limitations in this study. First, this was a retrospective study. Second, small SMTs often tended to undergo MCB, and MCB was rarely tried for extraluminal SMTs. Thus, there was selection bias in this study. Third, the sample size was relatively small in this study, especially in the MCB group.

In conclusion, MCB has the advantage of enabling immunohistological diagnosis, and MCB is good for obtaining specimens of small SMTs. In addition, MCB is non-inferior in other points compared to EUS-FNA. MCB may be an effective procedure for diagnosing gastric SMT, especially for small-size SMTs located in the middle third of the stomach.

\section{Acknowledgement}

We thank Dr. Hisashi Tateyama and Dr. Megumi Yoshida for their advice about histopathological findings. 


\section{Case Reports in Gastroenterology}

\begin{tabular}{l|l} 
Case Rep Gastroenterol 2019;13:185-194 \\
\hline DOI: 10.1159/000499442 & $\begin{array}{l}\text { @ } 2019 \text { The Author(s). Published by S. Karger AG, Basel } \\
\text { www.karger.com/crg }\end{array}$
\end{tabular}

Adachi et al.: Efficacy of Mucosal Cutting Biopsy for the Histopathological Diagnosis of Gastric Submucosal Tumors

\section{Statement of Ethics}

These procedures were assessed by the responsible review committee on Human Experimentation (our institution and national) and Helsinki Declaration of 1964. Informed consent was obtained from all patients by opt-out system.

\section{Disclosure Statement}

The authors have no conflicts of interest to disclose.

\section{References}

1 Japan Society of Clinical Oncology, Japanese Gastric Cancer Association, Japanese Study Group on GIST. GIST Therapeutic Guidelines. Tokyo: Kanehara Co., Ltd; 2014.

2 Jhala NC, Jhala D, Eltoum I, Vickers SM, Wilcox CM, Chhieng DC, et al. Endoscopic ultrasound-guided fineneedle aspiration biopsy: a powerful tool to obtain samples from small lesions. Cancer. 2004 Aug;102(4):239-46.

3 Miettinen M, Sobin LH, Lasota J. Gastrointestinal stromal tumors of the stomach: a clinicopathologic, immunohistochemical, and molecular genetic study of 1765 cases with long-term follow-up. Am J Surg Pathol. 2005 Jan;29(1):52-68.

4 Matsui M, Goto H, Niwa Y, Arisawa T, Hirooka Y, Hayakawa T. Preliminary results of fine needle aspiration biopsy histology in upper gastrointestinal submucosal tumors. Endoscopy. 1998 Nov;30(9):750-5.

5 Mekky MA, Yamao K, Sawaki A, Mizuno N, Hara K, Nafeh MA, et al. Diagnostic utility of EUS-guided FNA in patients with gastric submucosal tumors. Gastrointest Endosc. 2010 May;71(6):913-9.

6 Wiech T, Walch A, Werner M. Histopathological classification of nonneoplastic and neoplastic gastrointestinal submucosal lesions. Endoscopy. 2005 Jul;37(7):630-4.

7 Wiersema MJ, Vilmann P, Giovannini M, Chang KJ, Wiersema LM. Endosonography-guided fine-needle aspiration biopsy: diagnostic accuracy and complication assessment. Gastroenterology. 1997 Apr;112(4):1087-95.

8 Akahoshi K, Sumida Y, Matsui N, Oya M, Akinaga R, Kubokawa M, et al. Preoperative diagnosis of gastrointestinal stromal tumor by endoscopic ultrasound-guided fine needle aspiration. World J Gastroenterol. 2007 Apr;13(14):2077-82.

9 Hoda KM, Rodriguez SA, Faigel DO. EUS-guided sampling of suspected GI stromal tumors. Gastrointest Endosc. 2009 Jun;69(7):1218-23.

10 Aso A, Ihara E, Kubo H, Osoegawa T, Oono T, Nakamura K, et al. Gastric gastrointestinal stromal tumor smaller than 20 mm with liver metastasis. Clin J Gastroenterol. 2013 Feb;6(1):29-32.

11 Tanaka J, Oshima T, Hori K, Tomita T, Kim Y, Watari J, et al. Small gastrointestinal stromal tumor of the stomach showing rapid growth and early metastasis to the liver. Dig Endosc. 2010 Oct;22(4):354-6.

12 Kataoka M, Kawai T, Yagi K, Sugimoto H, Yamamoto K, Hayama Y, et al. Mucosal cutting biopsy technique for histological diagnosis of suspected gastrointestinal stromal tumors of the stomach. Dig Endosc. 2013 May;25(3):274-80.

13 Ihara E, Matsuzaka H, Honda K, Hata Y, Sumida Y, Akiho H, et al. Mucosal-incision assisted biopsy for suspected gastric gastrointestinal stromal tumors. World J Gastrointest Endosc. 2013 Apr;5(4):191-6.

14 Ikehara H, Li Z, Watari J, Taki M, Ogawa T, Yamasaki T, et al. Histological diagnosis of gastric submucosal tumors: A pilot study of endoscopic ultrasonography-guided fine-needle aspiration biopsy vs mucosal cutting biopsy. World J Gastrointest Endosc. 2015 Oct;7(14):1142-9.

15 Akahoshi K, Oya M, Koga T, Koga H, Motomura Y, Kubokawa M, et al. Clinical usefulness of endoscopic ultrasound-guided fine needle aspiration for gastric subepithelial lesions smaller than $2 \mathrm{~cm}$. J Gastrointestin Liver Dis. 2014 Dec;23(4):405-12.

16 Sekine M, Imaoka H, Mizuno N, Hara K, Hijioka S, Niwa Y, et al. Clinical course of gastrointestinal stromal tumor diagnosed by endoscopic ultrasound-guided fine-needle aspiration. Dig Endosc. 2015 Jan;27(1):4452. 


\section{Case Reports in Gastroenterology}

Adachi et al.: Efficacy of Mucosal Cutting Biopsy for the Histopathological Diagnosis of Gastric Submucosal Tumors

17 Niimi K, Goto 0, Kawakubo K, Nakai Y, Minatsuki C, Asada-Hirayama I, et al. Endoscopic ultrasound-guided fine-needle aspiration skill acquisition of gastrointestinal submucosal tumor by trainee endoscopists: A pilot study. Endosc Ultrasound. 2016 May-Jun;5(3):157-64.

18 Fernández-Esparrach G, Sendino O, Solé M, Pellisé M, Colomo L, Pardo A, et al. Endoscopic ultrasoundguided fine-needle aspiration and trucut biopsy in the diagnosis of gastric stromal tumors: a randomized crossover study. Endoscopy. 2010 Apr;42(4):292-9.

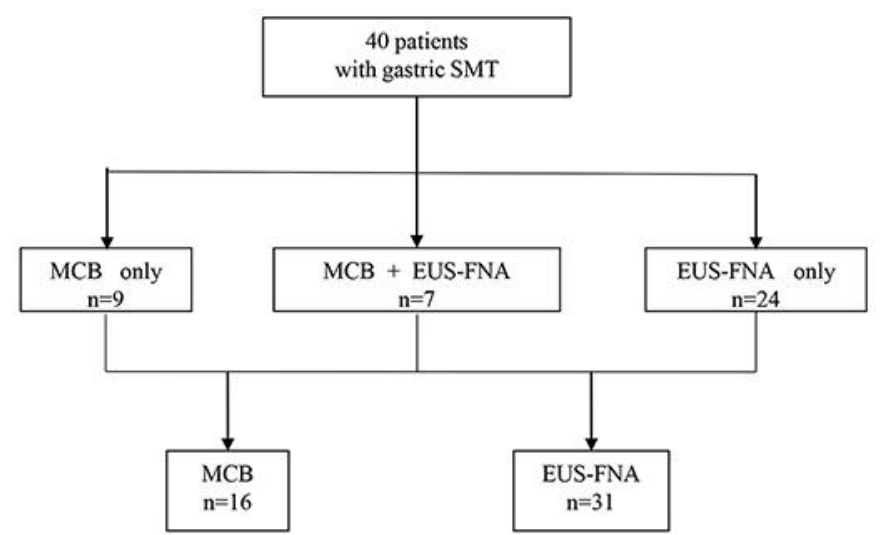

Fig. 1. Flow diagram of this study. MCB alone was performed in 9 patients, and EUS-FNA alone was performed in 24 patients. Both MCB and EUS-FNA were performed in 7 patients. Thus, 16 patients underwent MCB, and 31 patients underwent EUS-FNA. 


\section{Case Reports in Gastroenterology}

Case Rep Gastroenterol 2019;13:185-194
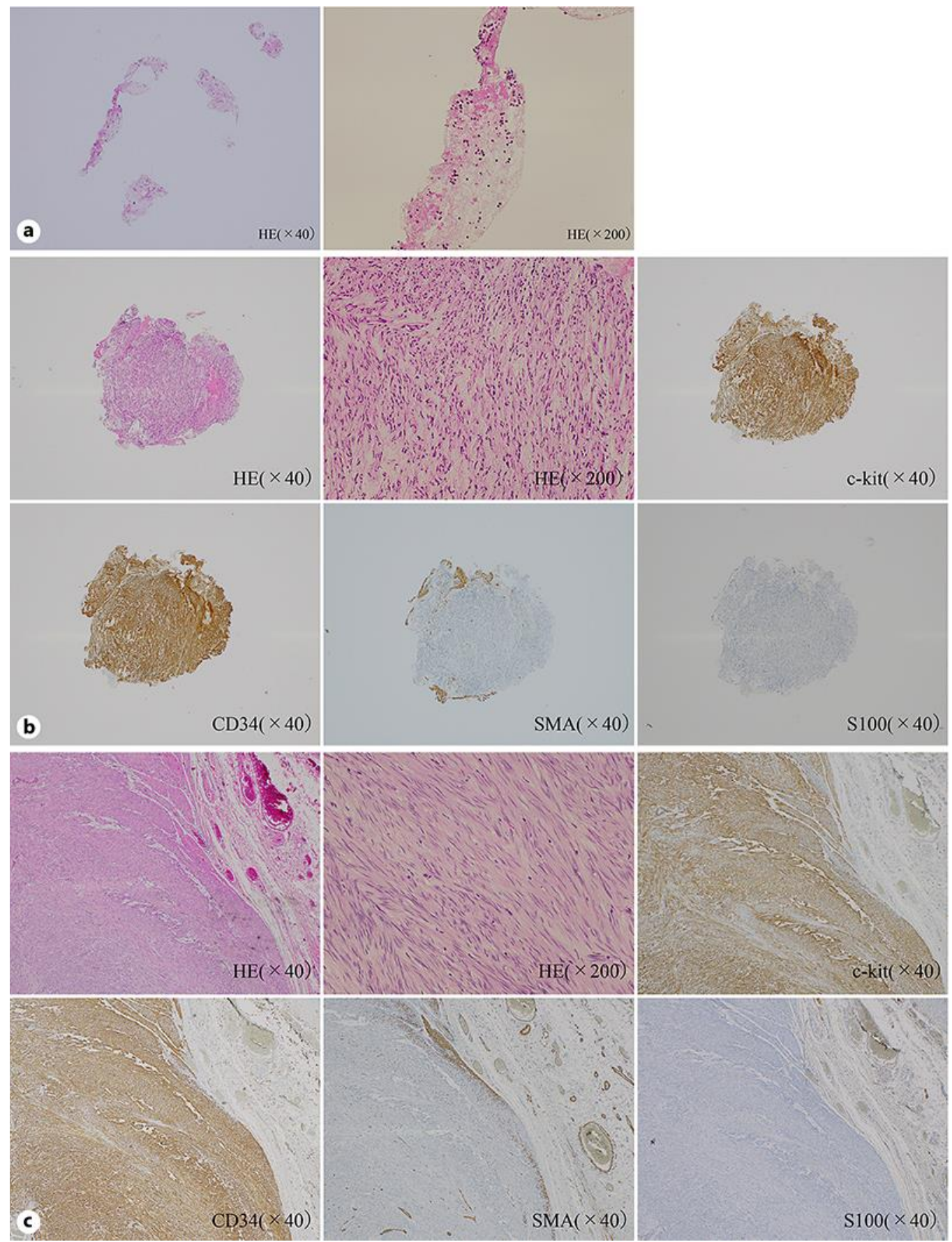


\section{Case Reports in Gastroenterology}

Fig. 2. a Histopathological finding in the EUS-FNA specimen. HE staining revealed only blood clot. Histopathological diagnosis including immunohistochemistry could not be made. $\mathbf{b}$ Histopathological examination in the MCB specimen. Biopsied specimen showing spindle cells (HE). Immunohistochemical staining was positive for c-kit and CD34 and negative for SMA and S100. So, this SMT was diagnosed as GIST. c Histopathological examination in the resected specimen. There are spindle cells that originated from muscle layer. Immunohistochemical staining was positive for c-kit and CD34 and negative for SMA and S100. This staining was completely the same as the specimen obtained by MCB.

Table 1. Summary of the patients with submucosal tumors who underwent mucosal cutting biopsy (MCB) and endoscopic ultrasonography-guided fine needle aspiration biopsy (EUS-FNA)

\begin{tabular}{llll}
\hline & MCB & EUS-FNA & $p$ value \\
\hline$n$ & 16 & 31 & \\
Age, years (mean \pm SD) & $62.5 \pm 11.9$ & $66.4 \pm 11.6$ & \\
Sex & & & \\
$\quad$ Male & 6 & 17 & NS \\
$\quad$ Female & 10 & 14 & \\
Tumor location (upper, & & & \\
$\quad$ middle, lower) & $5,9,2$ & $14,14,3$ & NS \\
Tumor size, mm (mean \pm SD) & $21.2 \pm 7.4$ & $36.2 \pm 22$ & NS \\
$\quad$ Tumor size & & & \\
$\quad \leq 20$ mm & 7 & 3 & $<0.05$ \\
$\quad>20$ mm & 9 & 28 & \\
Growth pattern & & & NS \\
$\quad$ Intraluminal & 14 & 19 & \\
$\quad$ Extraluminal & 2 & 12 & NS \\
$\quad$ Layer of origin & & & \\
$\quad$ Muscle layer & 14 & 29 & \\
$\quad$ Not muscle layer & 2 & 2 & \\
\hline
\end{tabular}


Table 2. The result of MCB and EUS-FNA

\begin{tabular}{|c|c|c|c|}
\hline & MCB & EUS-FNA & $p$ value \\
\hline$n$ & 16 & 31 & \\
\hline Procedure time, $\min ($ mean $\pm \mathrm{SD})$ & $40.7 \pm 14.9$ & $45.3 \pm 19.2$ & NS \\
\hline Number of technical successes & 14 & 25 & NS \\
\hline Number of biopsy specimens (mean \pm SD) & $6.6 \pm 2.2$ & $2.5 \pm 1.3$ & $<0.05$ \\
\hline Successful diagnostic rate & $14 / 16(88 \%)$ & $25 / 31(81 \%)$ & NS \\
\hline \multicolumn{4}{|l|}{ Successful diagnostic rate with immuno- } \\
\hline staining possible & $13 / 14 * 1(93 \%)$ & $17 / 29 * 1(59 \%)$ & $<0.05$ \\
\hline Diagnostic rate with cases less than $2 \mathrm{~cm}$ & $6 / 7(86 \%)$ & $2 / 3(67 \%)$ & NS \\
\hline Number of complications & 0 & 0 & NS \\
\hline \multicolumn{4}{|l|}{ Histological diagnosis } \\
\hline Gastrointestinal stromal tumor & 5 & 20 & \\
\hline Leiomyoma & 4 & 1 & \\
\hline Schwannoma & 2 & 1 & \\
\hline Aberrant pancreas & 2 & 2 & \\
\hline Malignant lymphoma & 1 & 1 & \\
\hline Not diagnosed & 2 & $6 * 3$ & \\
\hline Number of operations & $7 * 2$ & $24 * 3$ & \\
\hline \multicolumn{4}{|l|}{ Concordant rate of pathologic findings after } \\
\hline operation & $6 / 7(86 \%)$ & $19 / 24(79 \%)$ & NS \\
\hline
\end{tabular}

*1 Two cases of aberrant pancreas existed in each group. They are excluded because of needlessness of immunostaining. *2 All cases of GIST were resected surgically. One schwannoma was operated because of its big ulcer. One SMT diagnosed as leiomyoma was operated because another GIST existed in the same patient, but after operation, the SMT turned out to be GIST, too. *3 All cases of GIST were resected surgically. Two cases of GIST existed in the "not diagnosed" group, and they were diagnosed by MCB. One gastric cancer existed in the "not diagnosed" group and it was operated. One schwannoma was operated because of big size and lymphadenopathy around the stomach.

Table 3. Successful diagnostic rate of EUS-FNA divided by the tumor location

\begin{tabular}{|c|c|c|c|}
\hline & $\mathrm{L}$ & M & $\mathrm{U}$ \\
\hline Intraluminal & $50 \%(1 / 2)$ & $60 \%(6 / 10)$ & $100 \%(7 / 7)$ \\
\hline Extraluminal & $100 \%(1 / 1)$ & $100 \%(4 / 4)$ & $86 \%(6 / 7)$ \\
\hline
\end{tabular}

L, lower part; M, middle part; U, upper part. 